\title{
TELESSAÚDE BRASIL REDES E TELEODONTOLOGIA: RELATO DA EXPERIÊNCIA EM MATO GROSSO DO SUL
}

\author{
Telehealth Brazil Networks Program and Teledentistry: Experience Report of Mato Grosso do Sul, Brazil \\ Adélia Delfina da Motta Silva Correia'; Beatriz Figueiredo Dobashi'; Crhistinne Cavalheiro Maymone Gonçalves ${ }^{3}$; \\ Marcelo Nakaya Kanomata ${ }^{4}$; Valéria Regina Feracini Duenhas Monreal ${ }^{5}$; Euder Alexandre Nunes ${ }^{6}$
}

\begin{abstract}
Resumo Este artigo trata do relato da experiência da Teleodontologia no Programa Telessaúde Brasil Redes em Mato Grosso do Sul, gerido pela Secretaria de Estado de Saúde. São apresentados consolidados referentes a 2012, tanto de teleconsultorias quanto de ações de teleducação em Odontologia, revelando a Teleodontologia como potente ferramenta para o apoio à Saúde Bucal na Atenção Primária.

Palavras-chave: Odontologia, Teleodontologia, Teleconsultoria, Teleducação, Atenção Primária à Saúde.
\end{abstract}

\begin{abstract}
This article aims to share Teledentistry experience from Telehealth Brazil Networks Program in Mato Grosso do Sul, which is managed by the State Department of Health. It is presented consolidated data referring to 2012, representing Dentistry teleconsultings and Dentistry tele-education actions, revealing Teledentistry as a powerful tool to support the Oral Health in Primary Health Care.
\end{abstract}

Keywords: Dentistry, Teledentistry, Teleconsulting, Tele-education, Primary Health Care.

\section{Introdução}

Considerando-se a Portaria 2546', bem como a Portaria 2554, que instituiu o Componente de Informatização e Telessaúde Brasil Redes na Atenção Básica², integrado ao Programa Telessaúde Brasil Redes, é importante registrar que Mato Grosso do Sul encontra-se na perspectiva do movimento nacional em prol da atenção primária como ordenadora do cuidado, conforme preconizado pelo Decreto $7508^{3}$, que veio regulamentar a Lei no 8.080 , para dispor sobre a organização do Sistema
Único de Saúde (SUS), com foco nas regiões de saúde e na sua organização em Rede de Atenção à Saúde 4 .

Para isso, o Programa utiliza-se de tecnologias de informação e comunicação, realizando a teleassistência e a teleducação, sendo que o Estado de Mato Grosso do Sul (MS) faz parte desta rede desde 2010, instalado na Secretaria de Estado de Saúde.

De acordo com Haddad e Skelton-Macedo (2012), pode se afirmar que:

a Teleodontologia, como campo de conhecimento integrante à Telessaúde, vem crescendo nos anos

1. Odontóloga, Mestre em Saúde Coletiva, Coordenadora Estadual de Telessaúde, Secretaria de Estado de Saúde de Mato Grosso do Sul, Campo Grande, Mato Grosso do Sul, Brasil; 2. Médica Sanitarista, Ex-Secretária de Estado de Saúde de Mato Grosso do Sul, Campo Grande, Mato Grosso do Sul, Brasil; 3. Odontóloga, Doutora em Saúde Pública, Universidade Federal da Grande Dourados, Dourados, Mato Grosso do Sul, Brasil; 4. Odontólogo, Especialista em Saúde da Família, Secretaria Municipal de Saúde Pública de Campo Grande, Campo Grande, Mato Grosso do Sul, Brasil; 5. Fonoaudióloga, Especialista em Gestão da Clínica nas Redes de Atenção à Saúde, Gerente Administrativo de Telessaúde, Secretaria de Estado de Saúde de Mato Grosso do Sul, Campo Grande, Mato Grosso do Sul, Brasil; 6 . Analista de Sistemas, Responsável Técnico de TI da Telessaúde, Secretaria de Estado de Saúde de Mato Grosso do Sul, Mato Grosso do Sul, Brasil. 
recentes, em especial com ênfase na teleducação interativa, na teleassistência e na produção de pesquisas multicêntricas (p.173)

Este trabalho apresenta ações em Teleodontologia, ligadas, basicamente, à teleassistência e à teleducação, do Núcleo do Programa Telessaúde Brasil Redes em Mato Grosso do Sul, no ano de 2012.

\section{Teleconsultorias em Odontologia em Mato Grosso do Sul}

De acordo com a Portaria 2546, existem dois tipos de Teleconsultoria': síncrona (online), quando a comunicação acontece em tempo real, e assíncrona (offline), quando a resposta não é simultânea, mas posterior via sistema (email ou software específico). Alkmin et al. (2008), lembram, entretanto, que, no Brasil, prevalecem as teleconsultorias assíncronas, até porque ainda há problemas em regiões remotas com acesso à banda larga de internet ${ }^{6}$.

Foi feito consolidado dos dados registrados de teleconsultorias executados pelo núcleo, ligados à saúde bucal. Ao final de 2012, havia 1.529 profissionais de saúde cadastrados no Sistema de Teleconsultoria (cedido pelo Hospital das Clínicas, da Universidade Federal de Minas Gerais /HC-UFMG), sendo 143 cirurgiões-dentistas (CD) e 41 auxiliares de saúde bucal (ASB). São dois os teleconsultores na área, um especialista em Saúde da Família e outro em Odontologia para Pacientes com Necessidades Especiais e Odontopediatria.

Até novembro de 2012, foram demandadas 205 dúvidas que geraram 319 teleconsultorias no sistema, sendo 20 destas em odontologia, que ensejaram 44 teleconsultorias, sobre temas como: processo de trabalho da saúde bucal na saúde da família, diagnóstico de alterações na boca e face, uso de medicamentos, toxicologia do flúor, manejo de doença periodontal em portadores de diabetes.

Há, ainda, dúvidas médicas que dizem respeito à saúde bucal, inclusive, com um caso que, a partir de fotos anexadas ao sistema, proporcionou o apoio ao diagnóstico diferencial de sarcoidose e neoplasia em lábio superior, com o devido encaminhamento na rede.

\section{Teleducação em Odontologia em Mato Grosso do Sul}

Na teleducação (conferências, aulas e cursos, ministrados por meio da utilização das tecnologias de informação e comunicação'), foram realizados 37 seminários virtuais, sendo 8 deles referentes à Saúde Bucal, com os temas: Saúde Bucal na Estratégia de Saúde da Família; Visita Domiciliar na Saúde Bucal; Hipertensão Arterial e Saúde Bucal; Diabetes na Saúde Bucal; Indicadores de Saúde Bucal no Programa Nacional de Melhoria do Acesso e da Qualidade da Atenção Básica; Saúde Bucal do Bebê; Centro de Especialidades Odontológicas (CEO) e Laboratório Regional de Prótese Dentária (LRPD). É importante registrar que muitos dos temas foram demandados pelas próprias equipes e que os seminários são gravados e disponibilizados no site do programa para reuso pelos profissionais que não puderam participar em tempo real (http://telessaude.saude.ms.gov.br/ portal/includes/videos/login.php).

\section{Comentários Finais}

O uso de recursos de Educação a Distância (EAD) no século XXI precisa ser encarado como forma de ampliação do acesso à educação e, no caso da saúde, que inclui a Odontologia, às melhores evidências disponíveis e não como precarização da educação, inclusive porque é completamente possível hoje usar desses recursos para exercícios práticos ${ }^{7}$, apresentando-se como potente fer- 
ramenta de educação permanente para alcançarmos o objetivo do SUS conforme seus princípios, consolidando as Redes de forma coerente, oportuna e acolhedora.

Assim, a Teleodontologia tem se mostrado uma importante ferramenta assistencial e educativa para o apoio aos profissionais de Saúde Bucal na Atenção Primária, em MS.

\section{Referências}

1. Ministério da Saúde (BR). Portaria n².546, de 27 de outubro de 2011. Redefine e amplia o Programa Telessaúde Brasil, que passa a ser denominado Programa Nacional Telessaúde Brasil Redes (Telessaúde Brasil Redes). 2011. http://www.saude.mt.gov.br/ upload/legislacao/2546-[5054-041111-SES-MT].pdf. Acesso: 30/10/2011.

2. Ministério da Saúde (BR). Portaria n².554, de 28 de outubro de 2011.Institui, no Programa de Requalificação de Unidades Básicas de Saúde, o Componente de Informatização e Telessaúde Brasil Redes na Atenção Básica, integrado ao Programa Nacional de Telessaúde Brasil Redes.2011. http://bvsms.saude.gov.br/bvs/saudelegis/gm/2011/ prt2554_28_10_2011.html. Acesso: 30/10/2011.
3. Governo Federal (BR). Decreto $n^{\circ} 7.508$, de 28 de junho de 2011. Regulamenta a Lei n 8.080, de 19 de setembro de 1990, para dispor sobre a organização do Sistema Único de Saúde - SUS, o planejamento da saúde, a assistência à saúde e a articulação interfederativa, e dá outras providências. 2011. http://www.jusbrasil.com.br/ legislacao/1028206/decreto-7508-11. Acesso: 15/07/2013.

4. Correia ADMS, Dobashi BF, Gonçalves CCM, AE, Duenhas,VFD. Telessaúde Brasi Redes em Mato Grosso do Sul. In: Mathias I, Monteiro A. Gold book [recurso eletrônico]: inovação tecnológica em educação e saúde. - Dados eletrônicos. - Rio de Janeiro: EdUERJ; 2012. p.241-254. http://www.telessaude.uerj.br/resource/goldbook/ pdf/15.pdf. Acesso: 15/07/2013.

5. Haddad AE, Skelton-Macedo MC. Teleodontologia na formação dos profissionais de saúde. In: Mathias I, Monteiro A. Gold book [recurso eletrônico] : inovação tecnológica em educação e saúde. - Dados eletrônicos. - Rio de Janeiro: EdUERJ; 2012. p.173-206. http://www.telessaude.uerj.br/resource/goldbook/pdf/12.pdf. Acesso: $15 / 07 / 2013$.

6. Alkmim MBM, Maia JX, Marcolino MS, Cunha LR, SILVA, GAC, Figueira RM, Ribeiro AL. Nove Anos de Experiência em Teleconsultorias para Atenção Primária. In: Mathias I, Monteiro A. Gold book [recurso eletrônico]: inovação tecnológica em educação e saúde. - Dados eletrônicos. - Rio de Janeiro: EdUERJ; 2012. p.224-240. http://www.telessaude.uerj.br/resource/goldbook/pdf/14.pdf. Acesso: 10/07/2013.

7. Costa MA. Educação a distância em saúde: um exercício epistemológico na aproximação destas áreas. Congresso da Associação Brasileira de Educação a Distância. 2009 Maio. Niterói, Rio de Janeiro, Brasil. 2009. http://www.abed.org.br/congresso2009/CD/trabalhos/1452009203611.pdf. Acesso: 15/07/2013. 